NCU-HEP-k092

Dec 2021

rev. Mar 2022

ed. Jul 2022

\title{
Mixed State Parametrization and Two-qubit Entanglement
}

\author{
Otto C. W. Kong* and Hock King Ting \\ Department of Physics and Center for High Energy and High Field Physics, \\ National Central University, Chung-Li 32054, Taiwan \\ ${ }^{*}$ Corresponding author. E-mail : otto@phy.ncu.edu.tw
}

\begin{abstract}
A generic scheme for the parametrization of mixed state systems is introduced. A slightly modified version specially for bipartite systems is then given, and especially applied to a twoqubit system. Various features of two-qubit entanglement are analyzed based on the scheme. Our formulation of the parametrization and the analysis of entanglement properties exploit the interplay between pure states as Hilbert space vectors and pure as well as mixed states as density matrices. Explicit entanglement results are presented, in terms of negativity and concurrence, for all two-qubit mixed states with one single parameter/coordinate among the full set of fifteen being zero and a few other interesting cases.
\end{abstract}

PACS numbers: 


\section{INTRODUCTION}

Entanglement, as the characteristic feature of quantum physics and a great resource for quantum information technology, is at the heart of modern interest in physical science and technology [1 3]. However, more than thirty years after the paper by Werner [4], which effectively kick-started a very active period of studies in various aspects of quantum entanglement, especially for mixed states, the 'simple' notion of a complete characterization of the simplest system with entanglement, namely a system of two-qubits, has not been available. We still do not have a general expression that gives the separability condition of a generic two-qubit mixed state in a single parametric form, not to say a general expression for even one measure of entanglement for any such inseparable states.

We present here an approach to the complete parametrization of any pure or mixed states of any finite-dimensional quantum system, which is an extension of that for the pure states to general mixed states, each with the pure state as the one among the class that is closest to the mixed state. It is then applied, with a small adjustment, to the case of bipartite systems, with the dominating pure state part as a generally entangled extension of that of a product state which is again closest to it.

As an alternative to parametrizations of mixed states or coordinations of the geometric

space of all of them for a quantum system [1, 5], our approach is marked by using strongly the links between pure states as normalized vectors in the corresponding Hilbert spaces and mixed states as their eigenensemble of pure states from their purification as pure states of a doubled system. It has advantages in connection with those links. In particular, we illustrate that through the application to the two-qubit case, for which we get entanglement results of all mixed states with one single parameter/coordinate among the full set of fifteen being zero. Results for a few other limiting cases are also presented. We have interesting results on explicit separability conditions and entanglement measures apparently not available in the literature. Our scheme, however, is more than just an alternative. It can be easily seen that the scheme incorporates some physically important parameters into the full coordination of the space of mixed states, hence is also wells suited for analyzing the geometric structure of the latter and its relationship with the entanglement features.

Apart from individual results, we illustrate some interesting features on how the entanglements of parts of a pure or mixed state contribute to the overall entanglement, with 
interference of entanglements in the pure states and (partial) cancellation of entanglements in the mixed states. The last has been generally appreciated in the notion of robustness

of entanglement [6]. As the positive partial transpose (PPT) condition is necessary and sufficient for separability of two-qubit states [7, 8], we use the negativity [9, 10], as a natural measure of PPT violation, as well as the perhaps more popular concurrence [11, 12] to study the entanglement. The two measures have interesting similarities and differences in their dependence on the basic parameters. The results are expected to be illustrating features that are in a way generic for systems with entanglement. They, in a way, complement the studies in Refs.[12, 13].

\section{AN APPROACH TO PARAMETRIZATION OF MIXED STATES}

\section{A. A Generic Parametrization of Mixed States for a Quantum System}

The general expression for a single qubit mixed state can be written as

$$
\begin{aligned}
\frac{1+r}{2}|\phi\rangle\langle\phi|+\frac{1-r}{2} & \left|\phi_{\perp}\right\rangle\left\langle\phi_{\perp}\left|=\left(\frac{1+r}{2}|c|^{2}+\frac{1-r}{2}|s|^{2}\right)\right| 0\right\rangle\langle 0| \\
+ & \left(\frac{1+r}{2}|s|^{2}+\frac{1-r}{2}|c|^{2}\right)|1\rangle\langle 1|+r \bar{s} c| 0\rangle\langle 1|+r \bar{c} s| 1\rangle\langle 0|,
\end{aligned}
$$

where $|\phi\rangle=c|0\rangle+s|1\rangle,\left|\phi_{\perp}\right\rangle=-\bar{s}|0\rangle+\bar{c}|1\rangle, c \equiv \cos \left(\frac{\theta}{2}\right) e^{\frac{-i \psi}{2}}$ and $s \equiv \sin \left(\frac{\theta}{2}\right) e^{\frac{i \psi}{2}}, 0 \leq \theta<\pi$, $0 \leq \psi<2 \pi$. with $0 \leq r \leq 1$ a measure of the purity of the state. The generic pure state $|\phi\rangle$ is given by a unitary transformation acting on the fixed state $|0\rangle$, as $U|0\rangle$, taking the $U$ as an operator, the matrix form of which is given by $\left(\begin{array}{cc}c & -\bar{s} \\ s & \bar{c}\end{array}\right)$. And $\left|\phi_{\perp}\right\rangle=U|1\rangle$; all state vectors normalized. It is important to note that while the full symmetry on the Hilbert space is an $S U(2)$, which is a three-parameter group, $U$ lives only in a subgroup of two parameters. There is a nontrivial little group for $|0\rangle$ which does not change the vector. We elaborate on that because the structure is what we will use repeatedly below to get the general parametrization, and something that has been overlooked in some of the literature.

We can write a generic pure two-qubit state as $|\Psi\rangle=q_{+}\left|\phi \phi^{\prime}\right\rangle+e^{i \zeta} q_{-}\left|\phi_{\perp} \phi_{\perp}^{\prime}\right\rangle$, or explicitly with the six real independent parameters as

$$
q_{+}\left(c c^{\prime}|00\rangle+c s^{\prime}|01\rangle+s c^{\prime}|10\rangle+s s^{\prime}|11\rangle\right)+e^{i \zeta} q_{-}\left(\bar{s} \bar{s}^{\prime}|00\rangle-\bar{s} \bar{c}^{\prime}|01\rangle-\bar{c} \bar{s}^{\prime}|10\rangle+\bar{c} \bar{c}^{\prime}|11\rangle\right)
$$


where $q_{ \pm}=\sqrt{\frac{1 \pm r}{2}}$. The reduced density matrix $\rho_{A}$ is exactly given by our expression of the general mixed state density matrix for the single qubit system; $\rho_{B}$ is of the same form with $c$ and $s$ replaced by $c^{\prime}$ and $s^{\prime}$. We note that the concurrence [11], as the essentially unique measure of the entanglement of the state, is given by $\mathcal{C}_{p}=2 q_{+} q_{-}$, and $q_{+}^{2}$ and $q_{-}^{2}$ are the eigenvalues of the corresponding density matrix $\rho_{A B}$. Moreover, among the six parameters in $|\Psi\rangle, r$ uniquely characterizes the entanglement and hence it is nonlocal. The phase parameter $\zeta$ is not exactly local as it does not belong to one of the two qubits, as the rest four. However, its value can be changed with a phase transformation on $\left|\phi_{\perp}\right\rangle$ or one on $\left|\phi_{\perp}^{\prime}\right\rangle$. The $3 \times 2=6$ parameter set of local $S U(2)$ transformations only affects the values of 5 out of the 6 parameters/coordinates in the description of the generic pure two-qubit state.

For a generic mixed state, one has a purification of it [1] in the sense that it can always be obtained as the reduced density matrix of a pure state of a bipartite system. We start from the Schmidt decomposition for the latter given as

$$
\sum_{k=0}^{N-1} \sqrt{\mu_{k}}\left|e_{k}\right\rangle\left|e_{k}^{\prime}\right\rangle,
$$

$0 \leq \sqrt{\mu_{k}} \leq 1, \sum_{k=0}^{N-1} \mu_{k}=1$, with $\left|e_{k}\right\rangle$ and $\left|e_{k}^{\prime}\right\rangle$ being orthonormal sets of the two component Hilbert spaces having the same dimension $n$. To be definite, an ordering of the labeling should be specified, and we generally take one such that the sequence of $\mu_{k}$ is a decreasing one. We can express all $\left|e_{k}\right\rangle$ and $\left|e_{k}^{\prime}\right\rangle$ for an arbitrary pure state in terms of unitary transformations from a fixed basis. It is straightforward to give a coordination of the pure state in terms of coordinates for the $\left|e_{k}\right\rangle$ and $\left|e_{k}^{\prime}\right\rangle$. The above expression for the two-qubit pure state of is one given by $\mu_{0}=q_{+}^{2}$ and $\mu_{1}=q_{-}^{2}$. Note that we have to allow the relative phase $e^{i \zeta}$ as we have arbitrarily fixed the phase in $\left|\phi_{\perp} \phi_{\perp}^{\prime}\right\rangle$. Retrieving the mixed state as reduced density matrix of the purification gives us the coordination of the space of mixed state $\mathcal{M}$. The strategy works for any quNit system, i.e. system with an $N$-dimensional Hilbert space, though the number of coordinates of course grows fast with $N$.

Let us give an explicit description for such a coordination/parametrization of qutrit states. From the Schmidt decomposition, we first express $\left|e_{0}\right\rangle$ as a unitary transform of $|0\rangle$, up to an overall phase factor. The little group is $S U(2)$ acting on the orthogonal subspace, and we can take away an overall phase factor. Hence we need only $8-3-1=4$ parameters. 
The transform we denote by $U_{20}$ given as the explicit matrix

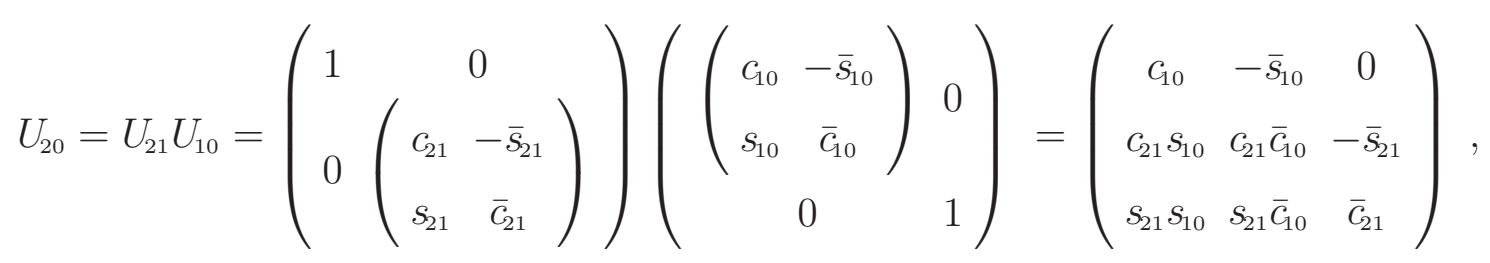

adopting the general relations of $c_{. .}=e^{\frac{-i}{2} \psi_{. .}} \cos \frac{1}{2} \theta_{. .}$and $s_{. .}=e^{\frac{i}{2} \psi_{* .}} \sin \frac{1}{2} \theta_{. .}$, where .. represents any one of the composite indices. Dropping all the phases, the column vector of the matrix, and its higher-dimensional analogs, as a generic orthonormal basis of a real vector space coordinate has been widely used. The phases $\psi$.. gives the relative phases between the corresponding components. Generalization to arbitrary $N$ is obvious, i.e. one can take the fixed vector $|0\rangle$ to an arbitrary (normalized) one, in an $n$-dimensional Hilbert, up to an overall phase factor, space by a

$$
U_{(n-1) 0}=U_{(n-1)(n-2)} U_{(n-2)(n-3)} \cdots U_{10}
$$

with $2(n-1)$ parameters. The little group is $S U(n-1)$. After writing $\left|e_{0}\right\rangle$ as $U_{20}|0\rangle$, and similarly $\left|e_{0}^{\prime}\right\rangle$ as $U_{20}^{\prime}|0\rangle$, we note that $\left|e_{1}\right\rangle$ and $\left|e_{2}\right\rangle$ live in a Hilbert space spanned by $U_{20}|1\rangle$, and $U_{20}|2\rangle$. We can simply repeat the strategy to write $\left|e_{1}\right\rangle$, up to an overall phase factor, as $\tilde{U}_{21} U_{20}|1\rangle$ through a new $\tilde{U}_{21}$. $\tilde{U}_{21} U_{20}|2\rangle$ then has to be $\left|e_{2}\right\rangle$ up to a phase factor. Hence, a generic two-qutrit pure state can be written as

$$
\sqrt{\mu_{0}} U_{20}|0\rangle \otimes U_{20}^{\prime}|0\rangle+\sqrt{\mu_{1}} e^{i \zeta_{1}} \tilde{U}_{21} U_{20}|1\rangle \otimes \tilde{U}_{21}^{\prime} U_{20}^{\prime}|1\rangle+\sqrt{\mu_{2}} e^{i \zeta_{2} 1} \tilde{U}_{21} U_{20}|2\rangle \otimes \tilde{U}_{21}^{\prime} U_{20}^{\prime}|2\rangle
$$

where we have put in the relative phases $e^{i \zeta_{1}}$ and $e^{i \zeta_{2}}$. A word of caution in the reading of our naive operator notation of the unitary transformations is in order here. The $\tilde{U}_{21}$, and similarly $\tilde{U}_{21}^{\prime}$, is a unitary transformation acting only within the vector subspace spanned by $U_{20}|1\rangle$ and $U_{20}|2\rangle$. While it has the general form of $S U(2)$ matrix adopted here on the basis of the two vectors, putting it in the form of the $U_{21}$ as a $3 \times 3$ matrix would be wrong. $\tilde{U}_{21}$ does not act on $\left|e_{0}\right\rangle=U_{20}|0\rangle$ the coordinate vector of which is given by the first column of $U_{20}$. (The right matrix product is to be taken in reverse order, in the original basis.)

To get back to single-qutrit mixed states, we simply have to take the reduced density matrix. We have

$$
\mu_{0} U_{20}|0\rangle\left\langle 0\left|U_{20}^{\dagger}+\mu_{1} \tilde{U}_{21} U_{20}\right| 1\right\rangle\left\langle 1\left|U_{20}^{\dagger} \tilde{U}_{21}^{\dagger}+\mu_{2} \tilde{U}_{21} U_{20}\right| 2\right\rangle\langle 2| U_{20}^{\dagger} \tilde{U}_{21}^{\dagger}
$$


as a generic qutrit state realized in terms of their eigenensembles. The eigenvalues are exactly the $\mu_{k}$. In the kind of expressions as the above for the mixed state, one can think about the $U_{20}$ and $\tilde{U}_{21}$ type of symbols as referring to the operators content of which as illustrated by the matrix expression for $U_{20}$ given above. We have actually an expression for any mixed state, here for a qutrit, in terms of the proper number of independent parameters which can be seen as providing a coordinate system for the space of mixed states. We have here eight parameters, the $3 \times 2$ in $U_{21}, U_{10}$ and $\tilde{U}_{21}$ plus the two independent ones among $\mu_{k}$. A simple good choice (in general) is $1 \geq \nu_{i} \equiv i\left(\mu_{i-1}-\mu_{i}\right) \geq 0, i \neq 0$. The explicit real coordinates, in this case, are given by $\theta_{21}, \psi_{21}, \theta_{10}, \psi_{10}, \tilde{\theta}_{21}, \tilde{\psi}_{21}, \nu_{1}$, and $\nu_{2}$. However, for many purposes, using the $\mu_{k}$ set may be more convenient.

Similarly, one can give a generic mixed state for single qutetrait as

$$
\begin{aligned}
\mu_{0} U_{30}|0\rangle\langle 0| & U_{30}^{\dagger}+\mu_{1} \tilde{U}_{31} U_{30}|1\rangle\langle 1| U_{30}^{\dagger} \tilde{U}_{31}^{\dagger} \\
& +\mu_{2} \widetilde{U}_{32} \tilde{U}_{31} U_{30}|2\rangle\left\langle 2\left|U_{30}^{\dagger} \tilde{U}_{31}^{\dagger} \widetilde{U}_{32}^{\dagger}+\mu_{3} \widetilde{U}_{32} \tilde{U}_{31} U_{30}\right| 3\right\rangle\langle 3| U_{30}^{\dagger} \tilde{U}_{31}^{\dagger} \widetilde{U}_{32}^{\dagger}
\end{aligned}
$$

Note that $\widetilde{U}_{32}$ is a different operator from $\tilde{U}_{32}$ inside $\tilde{U}_{31}=\tilde{U}_{32} \tilde{U}_{21}$. One can easily extrapolate from here to the large $n$ cases and check that the approach does give the right answer for the dimensions of the pure state and mixed state spaces in general.

\section{B. Modified Parametrization for a Bipartite System}

Next, we turn to a more interesting case, that of mixed two-qubit states. A two-qubit system can, first of all, be seen as one of a single qutetrait. From the result for the twoqutetrait pure state to single qutetrait mixed state reduction, we can first take the identification $|0\rangle=|00\rangle,|1\rangle=|11\rangle,|2\rangle=|01\rangle$, and $|3\rangle=|10\rangle$, and replace $U_{30}|0\rangle$ by our earlier expression for a generic two-qubit pure state. It can indeed be easily appreciated that the latter can be seen as the result of a unitary transformation acting on $|00\rangle$. Explicitly, we have $U_{22}$ as an operator being given by $U_{q}\left(U_{\phi} \otimes U_{\phi^{\prime}}\right)$ where

$$
U_{q}=\left(\begin{array}{cc}
q_{+} & -e^{-i \zeta} q_{-} \\
e^{i \zeta} q_{-} & q_{+}
\end{array}\right)
$$

acts in the vector subspace spanned by the basis vector $\left|\phi \phi^{\prime}\right\rangle$ and its orthogonal complement $\left|\phi_{\perp} \phi_{\perp}^{\prime}\right\rangle$. One can have $q_{+}=\cos \chi, q_{-}=\sin \chi$, with $\cos 2 \chi=r, \chi$ being the Schmidt 
angle of the mixing for the single-qubit state. The transformation $U_{22}$ is to be adopted for the replacement of $U_{30}$ in the expression above. The trick enforces the six coordinates for the description of a pure two-qubit state as part of the full set of fifteen coordinates for the description of a generic one. More specifically, we take our four $\left|e_{k}\right\rangle$ states as $U_{22}|0\rangle$, $U_{31} U_{22}|1\rangle, \tilde{U}_{32} U_{31} U_{22}|2\rangle$, and $\tilde{U}_{32} U_{31} U_{22}|3\rangle$, respectively, and the parallel for four $\left|e_{k}^{\prime}\right\rangle$ states. Those parameters inside $U_{31}$ and $\tilde{U}_{32}$, and the three $\nu_{i}$ gives the extra nine coordinates needed to fully describe any mixed two-qubit state in the fifteen dimensional space. Explicitly, we have

$$
\begin{aligned}
\left|e_{0}\right\rangle & =U_{22}|0\rangle=|\Psi\rangle=q_{+}\left|\phi \phi^{\prime}\right\rangle+e^{i \zeta} q_{-}\left|\phi_{\perp} \phi_{\perp}^{\prime}\right\rangle \\
\left|e_{1}\right\rangle & =U_{31} U_{22}|1\rangle=U_{31}\left(-e^{-i \zeta} q_{-}\left|\phi \phi^{\prime}\right\rangle+q_{+}\left|\phi_{\perp} \phi_{\perp}^{\prime}\right\rangle\right) \\
& =c_{21}\left(-e^{-i \zeta} q_{-}\left|\phi \phi^{\prime}\right\rangle+q_{+}\left|\phi_{\perp} \phi_{\perp}^{\prime}\right\rangle\right)+s_{21}\left(c_{32}\left|\phi \phi_{\perp}^{\prime}\right\rangle+s_{32}\left|\phi_{\perp} \phi^{\prime}\right\rangle\right) \\
\left|e_{2}\right\rangle & =\tilde{U}_{32} U_{31} U_{22}|2\rangle=\tilde{U}_{32} U_{31}\left|\phi \phi_{\perp}^{\prime}\right\rangle=c_{0} U_{31}\left|\phi \phi_{\perp}^{\prime}\right\rangle+s_{0} U_{31}\left|\phi_{\perp} \phi^{\prime}\right\rangle \\
& =-c_{0} \bar{s}_{21}\left(-e^{-i \zeta} q_{-}\left|\phi \phi^{\prime}\right\rangle+q_{+}\left|\phi_{\perp} \phi_{\perp}^{\prime}\right\rangle\right)+\left(c_{0} \bar{c}_{21} c_{32}-s_{0} \bar{s}_{32}\right)\left|\phi \phi_{\perp}^{\prime}\right\rangle+\left(c_{0} \bar{c}_{21} s_{32}+s_{0} \bar{c}_{32}\right)\left|\phi_{\perp} \phi^{\prime}\right\rangle \\
\left|e_{3}\right\rangle & =\tilde{U}_{32} U_{31} U_{22}|3\rangle=\tilde{U}_{32} U_{31}\left|\phi_{\perp} \phi^{\prime}\right\rangle=-\bar{s}_{0} U_{31}\left|\phi \phi_{\perp}^{\prime}\right\rangle+\bar{c}_{0} U_{31}\left|\phi_{\perp} \phi^{\prime}\right\rangle \\
& =\bar{s}_{0} \bar{s}_{21}\left(-e^{-i \zeta} q_{-}\left|\phi \phi^{\prime}\right\rangle+q_{+}\left|\phi_{\perp} \phi_{\perp}^{\prime}\right\rangle\right)-\left(\bar{s}_{0} \bar{c}_{21} c_{32}+\bar{c}_{0} \bar{s}_{32}\right)\left|\phi \phi_{\perp}^{\prime}\right\rangle-\left(\bar{s}_{0} \bar{c}_{21} s_{32}-\bar{c}_{0} \bar{c}_{32}\right)\left|\phi_{\perp} \phi^{\prime}\right\rangle,
\end{aligned}
$$

where we have used simply $c_{0}$ and $s_{0}$ for the parameter in $\tilde{U}_{32}$. We may also use the notation $\left|\Psi_{\perp}\right\rangle \equiv U_{22}|1\rangle$. The expressions above is all we need to write down a generic two-qubit (mixed) state, and it shows the convenient use of orthonormal basis $|\mathbf{0}\rangle \equiv\left|\phi \phi^{\prime}\right\rangle,|\mathbf{1}\rangle \equiv\left|\phi_{\perp} \phi_{\perp}^{\prime}\right\rangle$, $|\mathbf{2}\rangle \equiv\left|\phi \phi_{\perp}^{\prime}\right\rangle$, and $|\mathbf{3}\rangle \equiv\left|\phi_{\perp} \phi^{\prime}\right\rangle$, hiding the four local parameters in $|\phi\rangle$ and $\left|\phi^{\prime}\right\rangle$. The effect of local, relative, phase transformations given by one on $\left|\phi_{\perp}\right\rangle$ or $\left|\phi_{\perp}^{\prime}\right\rangle$ has effects on the eleven parameters/coordinates explicitly shown in the expressions that takes a bit more effort to trace. We leave the full expression of the mixed two-qubit state density matrix to an appendix, for reference. We will refer to the basis as the bold-type basis below. It is a natural set of basis for the Hilbert state of the two-qubit pure states.

It is interesting to note that the scaled Hilbert-Schmidt distance [1] between an arbitrary state $\rho$ and $E_{\Psi} \equiv|\Psi\rangle\langle\Psi|$ is simply given by

$$
\begin{aligned}
D_{2}\left(\rho, E_{\Psi}\right) \equiv & \frac{1}{\sqrt{2}} D_{H S}\left(\rho, E_{\Psi}\right)=\sqrt{\frac{1-2 \operatorname{Tr}\left(\rho E_{\Psi}\right)+\operatorname{Tr} \rho^{2}}{2}} \\
& =\sqrt{\left(1-\mu_{0}\right)^{2}-\left(\mu_{1} \mu_{2}+\mu_{2} \mu_{3}+\mu_{3} \mu_{1}\right)}
\end{aligned}
$$


It is easy to see $E_{\Psi}$ is really the pure state that is the closest to $\rho$. Moreover, $S_{\Psi} \equiv|\mathbf{0}\rangle\langle\mathbf{0}|$ is the product state closest to the generally entangled pure state $E_{\Psi}$ at $D_{2}\left(S_{\Psi}, E_{\Psi}\right)=q_{-}$

[14]. These are the interesting features that are not available in the popular parametrization/coordination of $\mathcal{M}$. Apart from giving a clear and direct identification of the subspace of pure states (at $\nu_{1}=1, \nu_{2}=\nu_{3}=0$ ) of dimension six, it allows direct implementation of important entanglement analyses like the PPT condition, which can be conveniently performed in the bold-type basis.

What has been described above for the two-qubit case can again be extrapolated to other two-quNit cases. Of course with growth of the dimension of the Hilbert space, the number of parameters/coordinates required to describe all mixed states may make it far too tedious to apply analytically.

\section{SOME ENTANGLEMENT FEATURES AND INTERFERENCE OF ENTAN- GLEMENT}

\section{A. Entanglement Results and Relations for the Different Pure State Parts}

We are interested in applying our parametrization of two-qubit mixed states to look at entanglement features. Actually, there is something to learn from first looking at the pure states involved in the eigenensemble expression. The concurrence, or equivalently negativity, of a two-qubit pure state is essentially the sole measure of its entanglement. We have for $E_{\Psi}$ and $E_{\Psi_{\perp}}$ the concurrence $\mathcal{C}_{\Psi}=\mathcal{C}_{\Psi_{\perp}}\left(=\mathcal{C}_{p}\right)$. Consider $E_{e_{1}} \equiv\left|e_{1}\right\rangle\left\langle e_{1}\right|$. The state vector is a linear combination of two orthonormal (generally) entangled states $\left|\Psi_{\perp}\right\rangle$ and $\left|\Psi_{1}\right\rangle \equiv c_{32}\left|\phi \phi_{\perp}^{\prime}\right\rangle+s_{32}\left|\phi_{\perp} \phi^{\prime}\right\rangle$, cf. Eq.(66), with concurrences given by $\mathcal{C}_{p}=2 q_{+} q_{-}$and $\mathcal{C}_{\Psi_{1}}=2 s_{32} c_{32}$, respectively. For $E_{e_{1}}$, we have the concurrence

$$
\mathcal{C}_{e_{1}}=\left|2 q_{+} q_{-} e^{i \zeta} \bar{c}_{21}^{2}+2 c_{32} s_{32} \bar{s}_{21}^{2}\right|=\left|\mathcal{C}_{p} e^{i \zeta} \bar{c}_{21}^{2}+\mathcal{C}_{\Psi_{1}} \bar{s}_{21}^{2}\right|
$$

showing an interesting pattern of interference of entanglement, depending sensitively on the relative phases in the linear combination of the two parts. For fixed $\mathcal{C}_{p}, \mathcal{C}_{\Psi_{1}}$, and $\theta_{21}$, $\mathcal{C}_{e_{1}}$ attains its maximum and minimum at $e^{i\left(2 \psi_{21}+\zeta\right)}= \pm 1$, of $\left|\mathcal{C}_{p} \cos ^{2}\left(\frac{\theta_{21}}{2}\right) \pm \mathcal{C}_{\Psi_{1}} \sin ^{2}\left(\frac{\theta_{21}}{2}\right)\right|$, respectively. The asymmetric appearance of $e^{i \zeta}$ here is an artifact of our parametrization. If one writes $\left|\Psi_{\perp}\right\rangle$ as a linear combination of $\left|\phi \phi^{\prime}\right\rangle$ and $\left|\phi_{\perp} \phi_{\perp}^{\prime}\right\rangle$ in the same form as $\left|\Psi_{1}\right\rangle$, 
interference depending only on the relative phase between the two entangled parts would be obtained. Similarly, we can see the same behavior looking into $\mathcal{C}_{e_{2}}$. Explicitly, we have

$$
\mathcal{C}_{e_{2}}=\left|\mathcal{C}_{p} e^{i \zeta} \bar{c}_{0}^{2} s_{21}^{2}+2\left(\bar{c}_{0} c_{21} \bar{s}_{32}+\bar{s}_{0} c_{32}\right)\left(\bar{c}_{0} c_{21} \bar{c}_{32}-\bar{s}_{0} s_{32}\right)\right|
$$

where $\left|2\left(\bar{c}_{0} c_{21} \bar{s}_{32}+\bar{s}_{0} c_{32}\right)\left(\bar{c}_{0} c_{21} \bar{c}_{32}-\bar{s}_{0} s_{32}\right)\right|$ is just $\left|s_{0}\right|^{2}+\left|c_{0}\right|^{2}\left|c_{21}\right|^{2}$ times the concurrence of the normalized state vector $\left|\Psi_{2}\right\rangle$, as a linear combination of $\left|\phi \phi_{\perp}^{\prime}\right\rangle$ and $\left|\phi_{\perp} \phi^{\prime}\right\rangle$, cf. Eq.(6) , and the normalization factor $\sqrt{\left|s_{0}\right|^{2}+\left|c_{0}\right|^{2}\left|c_{21}\right|^{2}}$ is exactly the amplitude of the part of the normalized vector in $\left|e_{2}\right\rangle$. The rest in the concurrence expression is about the phases. Explicitly,

$$
\begin{gathered}
\left|\Psi_{2}\right\rangle=\frac{1}{\sqrt{\left|s_{0}\right|^{2}+\left|c_{0}\right|^{2}\left|\mathcal{C}_{21}\right|^{2}}}\left[\left(c_{0} \bar{c}_{21} c_{32}-s_{0} \bar{s}_{32}\right)\left|\phi \phi_{\perp}^{\prime}\right\rangle+\left(c_{0} \bar{c}_{21} s_{32}+s_{0} \bar{c}_{32}\right)\left|\phi_{\perp} \phi^{\prime}\right\rangle\right] \\
\mathcal{C}_{\Psi_{2}}=\frac{2\left|\left(\bar{c}_{0} c_{21} \bar{s}_{32}+\bar{s}_{0} c_{32}\right)\left(\bar{c}_{0} c_{21} \bar{c}_{32}-\bar{s}_{0} s_{32}\right)\right|}{\left|s_{0}\right|^{2}+\left|c_{0}\right|^{2}\left|c_{21}\right|^{2}}
\end{gathered}
$$

In exact parallel, we have

$$
\mathcal{C}_{e_{3}}=\left|\mathcal{C}_{p} e^{i \zeta} s_{0}^{2} s_{21}^{2}+2\left(s_{0} c_{21} \bar{c}_{32}+c_{0} s_{32}\right)\left(s_{0} c_{21} \bar{s}_{32}-c_{0} c_{32}\right)\right|
$$

with

$$
\begin{gathered}
\left|\Psi_{3}\right\rangle=\frac{1}{\sqrt{\left|c_{0}\right|^{2}+\left|s_{0}\right|^{2}\left|c_{21}\right|^{2}}}\left[-\left(\bar{s}_{0} c_{32} \bar{c}_{21}+\bar{c}_{0} \bar{s}_{32}\right)\left|\phi \phi_{\perp}^{\prime}\right\rangle-\left(\bar{s}_{0} s_{32} \bar{c}_{21}-\bar{c}_{0} \bar{c}_{32}\right)\left|\phi_{\perp} \phi^{\prime}\right\rangle\right] \\
\mathcal{C}_{\Psi_{3}}=\frac{2\left|\left(s_{0} c_{21} \bar{c}_{32}+c_{0} s_{32}\right)\left(s_{0} c_{21} \bar{s}_{32}-c_{0} c_{32}\right)\right|}{\left|c_{0}\right|^{2}+\left|s_{0}\right|^{2}\left|c_{21}\right|^{2}}
\end{gathered}
$$

Note that $\left|\Psi_{1}\right\rangle,\left|\Psi_{2}\right\rangle$, and $\left|\Psi_{3}\right\rangle$ are generally mutually not orthogonal, though they are orthogonal to $|\Psi\rangle$ and $\left|\Psi_{\perp}\right\rangle$.

A mixed state as a particular mixture of pure states can be seen to have a concurrence as the weighted average of the concurrence of the pure states in the mixture. However, a mixed state or a density matrix can be written in different ways as a mixture. The concurrence, as a general measure of entanglement, is to be taken as the convex roof or the minimum among all possible mixture expressions. Hence, our results for the concurrence of the four pure states $E_{k} \equiv\left|e_{k}\right\rangle\left\langle e_{k}\right|$ provide at least an upper bound for the concurrence of any mixed state of the two-qubit system, in terms of the eleven parameters. Entanglement features are independent of the purely local parameters in the expressions of $|\phi\rangle$ and $\left|\phi^{\prime}\right\rangle$. However, we will see interesting cases below where the entanglement within different parts of a mixture cancels against one another. That seems to be more the rule than the exception. Another measure of entanglement particularly useful for the two-qubit states is the negativity, as twice 
the magnitude of the single negative eigenvalue of the partial transpose. Recall that for pure states, there is only a single notion of entanglement and the concurrence and negativity are equal. The difference between the two results for the mixed state is particularly interesting. Note that the partial transpose, as a Hermitian matrix, has a characteristic polynomial with only real coefficients. The complexity of the matrix elements, from our parametrization, rests on the complex phases of four mixing parameters of the pure state vector $\left|e_{k}\right\rangle$ in terms of the states of the bold-type basis. The parameters can only show up in the PPT condition and the negativity, and, we expect, also the concurrence, in specific real combinations. We believe we have the answer for them in our results for the entanglement of the $\left|e_{k}\right\rangle$ states, i.e. as the $\mathcal{C}_{e_{1}}, \mathcal{C}_{e_{2}}$, and $\mathcal{C}_{e_{3}}$ above. In fact, it is likely that any entanglement results for the system can be expressed in terms of the ten generally independent parameters of $\nu_{i}, \mathcal{C}_{p}, \mathcal{C}_{\Psi_{i}}$, and $\mathcal{C}_{e_{i}}$.

\section{B. Entanglement Results for a Large Class of Two-qubit States}

The entanglement feature of a special class of the generic mixed two-qubit states with a single of the fifteen parameters being zero is easy to analyze. The results are of course interesting in themselves. We also see them as very illustrative of how the entanglement of the pure states in its eigenensemble work with, or more like against, one another in contributing to the entanglement of the mixed state in general. One may compare that with the naive upper bound of the weighted average, which simply gives, for the concurrence for example, $\mathcal{C}_{\rho} \leq \sum_{k} \mu_{k} \mathcal{C}_{e_{k}}$ for the two-qubit states. When $\theta_{21}=0$, i.e. $s_{21}=0$, the density matrix reduces to two $2 \times 2$ diagonal blocks, a form that maintains in its partial transpose. The PPT condition is then given by the non-negativity of the determinants of the diagonal blocks of the latter, which is the two inequalities

$$
\mu_{0} \mu_{1}+\left(\mu_{0}-\mu_{1}\right)^{2} q_{+}^{2} q_{-}^{2} \geq\left(\mu_{2}-\mu_{3}\right)^{2}\left|c_{0} \bar{c}_{21} s_{32}+s_{0} \bar{c}_{32}\right|^{2}\left|\bar{c}_{0} c_{21} \bar{c}_{32}-\bar{s}_{0} s_{32}\right|^{2}
$$

and

$$
\mu_{2} \mu_{3}+\left(\mu_{2}-\mu_{3}\right)^{2}\left|c_{0} \bar{c}_{21} s_{32}+s_{0} \bar{c}_{32}\right|^{2}\left|\bar{c}_{0} c_{21} \bar{c}_{32}-\bar{s}_{0} s_{32}\right|^{2} \geq\left(\mu_{0}-\mu_{1}\right)^{2} q_{+}^{2} q_{-}^{2} .
$$

The first is trivially satisfied as $\mu_{0} \mu_{1} \geq\left(\mu_{2}-\mu_{3}\right)^{2}$. The other inequality is a true condition, which is equivalent to

$$
4 \mu_{2} \mu_{3}+\left(\mu_{2}-\mu_{3}\right)^{2} \mathcal{C}_{\Psi_{2}}^{2} \geq\left(\mu_{0}-\mu_{1}\right)^{2} \mathcal{C}_{p}^{2}
$$


where we have used $\left|s_{0}\right|^{2}+\left|c_{0}\right|^{2}\left|c_{21}\right|^{2}=1$ as $\left|c_{21}\right|=1$. Note that for the case, $\left|e_{2}\right\rangle$ and $\left|e_{3}\right\rangle$ reduce to two orthonormal linear combinations of $\left|\phi \phi_{\perp}^{\prime}\right\rangle$ and $\left|\phi_{\perp} \phi^{\prime}\right\rangle$, hence $\mathcal{C}_{e_{2}}=\mathcal{C}_{\Psi_{2}}=\mathcal{C}_{\Psi_{3}}=\mathcal{C}_{e_{3}}$; also we have $\mathcal{C}_{e_{1}}=\mathcal{C}_{e_{0}}=\mathcal{C}_{p}$. Considering particularly the case of $\mu_{2}=\mu_{3}=0$, it gives $\left(\mu_{0}-\mu_{1}\right) \mathcal{C}_{p}$ as the negativity of the resulting density matrix. That measures the entanglement of the state $\mu_{0} E_{\Psi}+\mu_{1} E_{\Psi_{\perp}}$, or that part of the full state $\rho$. This is a direct contrast to the weighted sum of $\left(\mu_{0}+\mu_{1}\right) \mathcal{C}_{p}$. Similarly, one can see $\left(\mu_{2}-\mu_{3}\right) \mathcal{C}_{\Psi_{2}}$ as negativity of the other part, namely $\mu_{2} E_{e_{2}}+\mu_{3} E_{e_{3}}=\mu_{2} E_{\Psi_{2}}+\mu_{3} E_{\Psi_{3}}$. Directly checking the negativity of the general $\left(s_{21}=0\right)$ case, we get, for the entangled states violating the above inequality,

$$
\mathcal{N}_{s_{21}=0}=\sqrt{\left(\mu_{0}-\mu_{1}\right)^{2} \mathcal{C}_{p}^{2}+\left(\mu_{2}-\mu_{3}\right)^{2}\left(1-\mathcal{C}_{\Psi_{2}}^{2}\right)}-\left(\mu_{2}+\mu_{3}\right)
$$

This shows the interesting but complicated interplay between the entanglement of the parts, as illustrated by the values of the negativities (which is the same as the concurrences for the pure states), of the two parts here of a mixed state contributed to the full state. Calculating the concurrence is only a little bit more involved [11]. The result reads

$$
\mathcal{C}_{s_{21}=0}=\left(\mu_{0}-\mu_{1}\right) \mathcal{C}_{p}-\sqrt{\left(\mu_{2}-\mu_{3}\right)^{2} \mathcal{C}_{\Psi_{2}}^{2}+4 \mu_{2} \mu_{3}}
$$

We see that $\left(\mu_{0}-\mu_{1}\right) \mathcal{C}_{p}$ is also the concurrence for the state $\mu_{0} E_{\Psi}+\mu_{1} E_{\Psi_{\perp}}$. That suggests that the negativity and concurrence of a mixed state with an eigenensemble as weighted sum of two exact orthogonal complements of the same two basis pure states, as vectors of the Hilbert space, has the same value as the difference between the two parts. We note that the concurrence equals to the negativity and they are independent of $\mathcal{C}_{\Psi_{2}}$ when $\mu_{2}=\mu_{3}$. Another interesting special limit to check is when $\mu_{3}$ vanishes. For the concurrence, we have the simple result of $\left(\mu_{0}-\mu_{1}\right) \mathcal{C}_{p}-\mu_{2} \mathcal{C}_{\Psi_{2}}$. That of the negativity, explicitly as $\sqrt{\left(\mu_{0}-\mu_{1}\right)^{2} \mathcal{C}_{p}^{2}-\mu_{2}^{2} \mathcal{C}_{\Psi_{2}}^{2}+\mu_{2}^{2}}-\mu_{2}$, is a bit more complicated, as the $\mu_{k}$ parameters are not completely independent. In general, both the negativity and the concurrence results are increasing functions of all, the independent, $\nu_{i}$ parameters. And they have essentially the maximum value of $\mu_{0} \mathcal{C}_{p}$ which can attain unity.

In the $s_{21}=0$ case, we basically have the entanglement as having the $\left(\mu_{0}-\mu_{1}\right) \mathcal{C}_{p}$ from the dominating $\mu_{0} E_{e_{0}}+\mu_{1} E_{e_{1}}$ contribution, which in itself is the difference between the two parts, being further partially canceled by that of the $\mu_{2} E_{e_{2}}+\mu_{3} E_{e_{3}}$ part. Short of having the generic entanglement results for the two-qubit state, we look at the other limits to check plausible similar features of entanglement cancellation. In Table 1, the entanglement results 


\begin{tabular}{|c|c|c|c|c|c|c|c|c|}
\hline & $E_{\Psi}=E_{e_{0}}$ & $E_{\Psi_{1}}$ & $E_{\Psi_{2}}$ & $E_{\Psi_{3}}$ & $E_{e_{1}}$ & $E_{e_{2}}$ & $E_{e_{3}}$ & PPT condition for $\rho=\sum \mu_{k} E_{e_{k}}$ \\
\hline$s_{21}=0$ & $\mathcal{C}_{p}$ & $\mathcal{C}_{\Psi_{1}}$ & $\mathcal{C}_{\Psi_{2}}$ & $\mathcal{C}_{\Psi_{2}}$ & $\mathcal{C}_{p}$ & $\mathcal{C}_{\Psi_{2}}$ & $\mathcal{C}_{\Psi_{2}}$ & $4 \mu_{2} \mu_{3}+\left(\mu_{2}-\mu_{3}\right)^{2} \mathcal{C}_{\Psi_{2}}^{2} \geq\left(\mu_{0}-\mu_{1}\right)^{2} \mathcal{C}_{p}^{2}$ \\
\hline$c_{21}=0$ & $\mathcal{C}_{p}$ & $\mathcal{C}_{\Psi_{1}}$ & $\mathcal{C}_{\Psi_{1}}$ & $\mathcal{C}_{\Psi_{1}}$ & $\mathcal{C}_{\Psi_{1}}$ & $\mathrm{I}\left(\mathcal{C}_{p}, \mathcal{C}_{\Psi_{1}}\right)$ & $\mathrm{I}\left(\mathcal{C}_{p}, \mathcal{C}_{\Psi_{1}}\right)$ & $\begin{array}{c}\mu_{2}=\mu_{3}: 4 \mu_{0} \mu_{2}+\left(\mu_{0}-\mu_{2}\right)^{2} \mathcal{C}_{p}^{2} \geq\left(\mu_{1}-\mu_{2}\right)^{2} \mathcal{C}_{\Psi_{1}}^{2} \\
\text { and } \quad 4 \mu_{1} \mu_{2}+\left(\mu_{1}-\mu_{2}\right)^{2} \mathcal{C}_{\Psi_{1}}^{2} \geq\left(\mu_{0}-\mu_{2}\right)^{2} \mathcal{C}_{p}^{2}\end{array}$ \\
\hline$s_{32}=0$ & $\mathcal{C}_{p}$ & 0 & $\mathcal{C}_{\Psi_{2}}^{\prime}$ & $\mathcal{C}_{\Psi_{3}}^{\prime}$ & $\left|c_{21}\right|^{2} \mathcal{C}_{p}$ & $\mathrm{I}\left(\mathcal{C}_{p}, \mathcal{C}_{\Psi_{2}}^{\prime}\right)$ & $\mathrm{I}\left(\mathcal{C}_{p}, \mathcal{C}_{\Psi_{3}}^{\prime}\right)$ & $\mathcal{C}_{p}=0,\left(\mu_{2}=\mu_{3}\right.$ or $\left.c_{21}=0\right):$ satisfied \\
\hline$c_{32}=0$ & $\mathcal{C}_{p}$ & 0 & $\mathcal{C}_{\Psi_{2}}^{\prime}$ & $\mathcal{C}_{\Psi_{3}}^{\prime}$ & $\left|c_{21}\right|^{2} \mathcal{C}_{p}$ & $\mathrm{I}\left(\mathcal{C}_{p}, \mathcal{C}_{\Psi_{2}}^{\prime}\right)$ & $\mathrm{I}\left(\mathcal{C}_{p}, \mathcal{C}_{\Psi_{3}}^{\prime}\right)$ & $\mathcal{C}_{p}=0,\left(\mu_{2}=\mu_{3}\right.$ or $\left.c_{21}=0\right):$ satisfied \\
\hline$s_{0}=0$ & $\mathcal{C}_{p}$ & $\mathcal{C}_{\Psi_{1}}$ & $\mathcal{C}_{\Psi_{1}}$ & $\mathcal{C}_{\Psi_{1}}$ & $\mathrm{I}\left(\mathcal{C}_{p}, \mathcal{C}_{\Psi_{1}}\right)$ & $\mathrm{I}\left(\mathcal{C}_{p}, \mathcal{C}_{\Psi_{1}}\right)$ & $\mathcal{C}_{\Psi_{1}}$ & $\begin{array}{l}\mu_{1}=\mu_{2}: 4 \mu_{1} \mu_{3}+\left(\mu_{1}-\mu_{3}\right)^{2} \mathcal{C}_{\Psi_{1}}^{2} \geq\left(\mu_{0}-\mu_{1}\right)^{2} \mathcal{C}_{p}^{2} \\
\mathcal{C}_{21}=0: 4 \mu_{0} \mu_{2}+\left(\mu_{0}-\mu_{2}\right)^{2} \mathcal{C}_{p}^{2} \geq\left(\mu_{1}-\mu_{3}\right)^{2} \mathcal{C}_{\Psi_{1}}^{2} \\
\text { and } 4 \mu_{1} \mu_{3}+\left(\mu_{1}-\mu_{3}\right)^{2} \mathcal{C}_{\Psi_{1}}^{2} \geq\left(\mu_{0}-\mu_{2}\right)^{2} \mathcal{C}_{p}^{2}\end{array}$ \\
\hline$c_{0}=0$ & $\mathcal{C}_{p}$ & $\mathcal{C}_{\Psi_{1}}$ & $\mathcal{C}_{\Psi_{1}}$ & $\mathcal{C}_{\Psi_{1}}$ & $\mathrm{I}\left(\mathcal{C}_{p}, \mathcal{C}_{\Psi_{1}}\right)$ & $\mathcal{C}_{\Psi_{1}}$ & $\mathrm{I}\left(\mathcal{C}_{p}, \mathcal{C}_{\Psi_{1}}\right)$ & $\begin{array}{r}c_{21}=0: 4 \mu_{0} \mu_{3}+\left(\mu_{0}-\mu_{3}\right)^{2} \mathcal{C}_{p}^{2} \geq\left(\mu_{1}-\mu_{2}\right)^{2} \mathcal{C}_{\Psi_{1}}^{2} \\
\text { and } \quad 4 \mu_{1} \mu_{2}+\left(\mu_{1}-\mu_{2}\right)^{2} \mathcal{C}_{\Psi_{1}}^{2} \geq\left(\mu_{0}-\mu_{3}\right)^{2} \mathcal{C}_{p}^{2}\end{array}$ \\
\hline
\end{tabular}

TABLE I: Entanglement of the pure states at the various limits and some related entanglement results for the mixed state: The $\mathrm{I}(\cdot, \cdot)$ symbol denotes an entanglement as the interference of the two parts, i.e. as the magnitude of a normalized complex linear combination of the two entanglement measures. Note that in general, $\mathcal{C}_{e_{i}}=\mathrm{I}\left(\mathcal{C}_{p}, \mathcal{C}_{\Psi_{i}}\right)$. Recall that $\mathcal{C}_{p}=2 q_{+} q_{-}$and $\mathcal{C}_{\Psi_{1}}=2 s_{32} c_{32}$. We have also $\mathcal{C}_{\Psi_{2}}^{\prime}=\frac{2\left|c_{0} s_{0} c_{21}\right|}{\left|s_{0}\right|^{2}+\left|c_{0}\right|^{2}\left|c_{21}\right|^{2}}$ and $\mathcal{C}_{\Psi_{3}}^{\prime}=\frac{2\left|c_{0} s_{0} c_{21}\right|}{\left|c_{0}\right|^{2}+\left|s_{0}\right|^{2}\left|c_{21}\right|^{2}}$. In each of the above cases, the concurrence is given by the square root of the right-hand side of the PPT inequality minus that of the left-hand side, and the negativity is given by $\sqrt{f\left(\mathcal{C}_{p}^{2}, \mathcal{C}_{\Psi_{1}}^{2}\right)+\left(\mu_{h}-\mu_{k}\right)^{2}}-\left(\mu_{h}+\mu_{k}\right)$ with the PPT condition taken in the form $4 \mu_{h} \mu_{k} \geq f\left(\mathcal{C}_{p}^{2}, \mathcal{C}_{\Psi_{1}}^{2}\right)\left(\mu_{h} \geq \mu_{k}\right.$, i.e. $\left.h<k\right)$, when the PPT condition is violated.

for the various pure states involved are given at the various limits of the mixing parameters inside the pure states. One can see that the $c_{21}=0, c_{0}=0$, or $s_{0}=0$ cases all involve only the two basic pure state entanglement as $\mathcal{C}_{p}$ and $\mathcal{C}_{\Psi_{1}}$. With an extra condition imposed, for example $\mu_{2}=\mu_{3}$ for the $c_{21}=0$ case, we have explicit results of some interest. In particular, for the last case, the PPT condition is given by

$$
4 \mu_{0} \mu_{2}+\left(\mu_{0}-\mu_{2}\right)^{2} \mathcal{C}_{p}^{2} \geq\left(\mu_{1}-\mu_{2}\right)^{2} \mathcal{C}_{\Psi_{1}}^{2},
$$

and

$$
4 \mu_{1} \mu_{2}+\left(\mu_{1}-\mu_{2}\right)^{2} \mathcal{C}_{\Psi_{1}}^{2} \geq\left(\mu_{0}-\mu_{2}\right)^{2} \mathcal{C}_{p}^{2}
$$


The negativity is

$$
\begin{aligned}
& \mathcal{N}_{c_{21}=0, \mu_{2}=\mu_{3}}=\sqrt{\left(\mu_{1}-\mu_{2}\right)^{2} \mathcal{C}_{\Psi_{1}}^{2}+\left(\mu_{0}-\mu_{2}\right)^{2}\left(1-\mathcal{C}_{p}^{2}\right)}-\left(\mu_{0}+\mu_{2}\right), \\
& \text { or } \mathcal{N}_{c_{21}=0, \mu_{2}=\mu_{3}}=\sqrt{\left(\mu_{0}-\mu_{2}\right)^{2} \mathcal{C}_{p}^{2}+\left(\mu_{1}-\mu_{2}\right)^{2}\left(1-\mathcal{C}_{\Psi_{1}}^{2}\right)}-\left(\mu_{1}+\mu_{2}\right),
\end{aligned}
$$

corresponding to the cases of the first and second inequalities above being violated, respectively. The matching concurrence is, respectively,

$$
\begin{aligned}
& \mathcal{C}_{c_{21}=0, \mu_{2}=\mu_{3}}=\left(\mu_{1}-\mu_{2}\right) \mathcal{C}_{\Psi_{1}}-\sqrt{\left(\mu_{0}-\mu_{2}\right)^{2} \mathcal{C}_{p}^{2}+4 \mu_{0} \mu_{2}}, \\
& \text { or } \mathcal{C}_{c_{21}=0, \mu_{2}=\mu_{3}}=\left(\mu_{0}-\mu_{2}\right) \mathcal{C}_{p}-\sqrt{\left(\mu_{1}-\mu_{2}\right)^{2} \mathcal{C}_{\Psi_{1}}^{2}+4 \mu_{1} \mu_{2}} .
\end{aligned}
$$

Here, depending on which entanglement of the key two parts dominate, i.e. if $\left(\mu_{0}-\mu_{2}\right) \mathcal{C}_{p}$ is smaller or larger than $\left(\mu_{1}-\mu_{2}\right) \mathcal{C}_{\Psi_{1}}$, it is only when the difference of the two parts is large enough that we have an entanglement that increases with $\mathcal{C}_{\Psi_{1}}$ and decreases with $\mathcal{C}_{p}$ or increases with $\mathcal{C}_{p}$ and decreases with $\mathcal{C}_{\Psi_{1}}$, respectively. That applies to both the negativity and the concurrence. The $\left(\mu_{0}-\mu_{2}\right) \mathcal{C}_{p}$ dominating case is an exact parallel with the $s_{21}=0$ case above. When $\left(\mu_{1}-\mu_{2}\right) \mathcal{C}_{\Psi_{1}}$ dominates, however, it is somewhat different. While the concurrence has the form $\mu_{1} \mathcal{C}_{\Psi_{1}}-\mu_{0} \mathcal{C}_{p}$ for $\mu_{2}=0$, and generally can still attain $\mu_{1} \mathcal{C}_{\Psi_{1}}$, its value is bounded by $\frac{1}{2}$ as the maximum $\mu_{1}$ from the $\mu_{k}$ hierarchy. The negativity has maximum in the form $\sqrt{\mu_{1}^{2} \mathcal{C}_{\Psi_{1}}^{2}+\mu_{0}^{2}}-\mu_{0}$ which, while still decreases with an increase in $\mu_{0}$, can only get to $\frac{\sqrt{2}-1}{2}$ at the maximum $\mu_{0}$ of $\frac{1}{2}$.

Other similar cases we have explicit entanglement results for are given in Table 1 . We refrain from presenting an explicit analysis of their basic features, which are much of a parallel to those discussed above.

\section{Applications to Generalizations of Werner States}

In terms of the scaled Hilbert-Schmidt geometry, the maximally mixed state $\left(\nu_{i}=0\right)$ and the lines connecting it at the center to the four corners of a tetrahedral (taken as the pure states $\left|e_{k}\right\rangle\left\langle e_{k}\right|$ here) is particularly interesting [1]. These are generalized Werner states [4, 14]. We write a generalized Werner state for our system as $x|\Phi\rangle\langle\Phi|+\frac{1-x}{4} I, x \in\left[-\frac{1}{3}, 1\right]$, where $|\Phi\rangle$ gives a pure state with any, not necessarily maximal, entanglement. We first take the case of $|\Phi\rangle$ being $\left|e_{0}\right\rangle$, i.e. $|\Psi\rangle$. We are really looking at the subspace $\mathcal{M}_{(13)}$ in Ref. [1], with $\mu \equiv \mu_{1}=\mu_{2}=\mu_{3}\left(\leq \frac{1}{4}\right)$, hence $\mu_{0}=1-3 \mu$. Any such mixed state clearly is described 
by the seven parameters/coordinates, including the six for the pure state of Eq.(2) and the mixing parameter $\mu$, or $\nu_{1}(=1-4 \mu)$ which is the $x$ in this case, except that no negative value of it is allowed. Explicitly, we have the line $W_{\mu} \equiv(1-4 \mu) E_{\Psi}+\mu I$. We want to check the separability of an even bigger set of states, one which has the same form of the density matrix in the bold-type basis but with no constraint on the $\mu_{k}$. We denote that by $W_{\vec{\mu}}$, which can be obtained by imposing $\theta_{32}=\theta_{21}=\theta_{0}=0$, hence $s_{32}=s_{21}=s_{0}=0$. Explicitly, $W_{\vec{\mu}}=\mu_{0} E_{\Psi}+\mu_{1} E_{\Psi_{\perp}}+\mu_{2}\left|\phi \phi_{\perp}^{\prime}\right\rangle\left\langle\phi \phi_{\perp}^{\prime}\left|+\mu_{3}\right| \phi_{\perp} \phi^{\prime}\right\rangle\left\langle\phi_{\perp} \phi^{\prime}\right|$. It is only a special case of the $s_{21}=0$ class we analyzed in the previous section. The separability condition is given by $\mathcal{C}_{p} \leq \frac{2 \sqrt{\mu_{2} \mu_{3}}}{\mu_{0}-\mu_{1}}$, which reduces to $\mathcal{C}_{p} \leq \frac{2 \mu}{1-4 \mu}=\frac{1-\nu_{1}}{2 \nu_{1}}$ for the generalized Werner states $W_{\mu}$. In fact, for the inseparable cases, we have the negativity

$$
\mathcal{N}_{W_{\vec{\mu}}}=\sqrt{\mathcal{C}_{p}^{2}\left(\mu_{0}-\mu_{1}\right)^{2}+\left(\mu_{2}-\mu_{3}\right)^{2}}-\left(\mu_{2}+\mu_{3}\right)
$$

and concurrence

$$
\mathcal{C}_{W_{\vec{\mu}}}=\left(\mu_{0}-\mu_{1}\right) \mathcal{C}_{p}-2 \sqrt{\mu_{2} \mu_{3}},
$$

hence $\mathcal{N}_{W_{\mu}}=\mathcal{C}_{W_{\mu}}=(1-4 \mu) \mathcal{C}_{p}-2 \mu=\nu_{1} \mathcal{C}_{p}-\frac{1-\nu_{1}}{2}$.

The separability condition for $W_{\mu}$ can be rewritten as $\left[2\left(2 \mathcal{C}_{p}-1\right) \mu-\mathcal{C}_{p}\right]\left[2\left(2 \mathcal{C}_{p}+1\right) \mu-\mathcal{C}_{p}\right] \leq$ 0 , which reduces to $\mu \geq \frac{\mathcal{C}_{p}}{2\left(2 \mathcal{C}_{p}+1\right)}$, or $\nu_{1} \leq \frac{1}{2 \mathcal{C}_{p}+1}$. Note that we have, for $\nu_{1}>\frac{1}{2 \mathcal{C}_{p}+1}$, $\mathcal{N}_{W_{\mu}}=\frac{\left(2 \mathcal{C}_{p}+1\right) \nu_{1}-1}{2}$. In particular, we have $\mu \geq \frac{1}{6}$ and $\nu_{1} \leq \frac{1}{3}$ for $\mathcal{C}_{p}=1$, and $\mu \geq \frac{1}{8}$ and $\nu_{1} \leq \frac{1}{2}$ for $\mathcal{C}_{p}=\frac{1}{2}$. For the limit of small $\mathcal{C}_{p}$, the separability condition tends to $\mu \geq \frac{\mathcal{C}_{p}}{2}$, or $\nu_{1} \leq 1-2 \mathcal{C}_{p}$ linear in $\mathcal{C}_{p}$. Of course for $\mathcal{C}_{p}=0$, all of $W_{\mu}$ are separable. One can easily obtain $D_{2}\left(W_{\mu}, E_{\Psi}\right)=\sqrt{6} \mu$. The result shows that one can consider the $W_{\mu}$ set as a straight line through $E_{\Psi}$ with $\mu$ as effectively a measure of distance [14], we have established clearly for any $\mathcal{C}_{p}$ value. The separable $W_{\mu}$ state closest to $E_{\Psi}$ is given by $\mu=\frac{\mathcal{C}_{p}}{2\left(2 \mathcal{C}_{p}+1\right)} \cdot{ }^{1}$ One can

1 The product state $S_{\Psi}$ as the particular $E_{\Psi}$ at $r=1$ is the product state that is closest to $E_{\Psi}$, as shown in Ref. 14], at a distance of $\sqrt{\frac{1-r}{2}}$. The maximally mixed state $W_{\frac{1}{4}}$ is at an $r$-independent distance of $\frac{\sqrt{6}}{4}$ from any $E_{\Psi}$, including $S_{\Psi}$. From simple trigonometry, the point on $W_{\mu}$ at the projection of $S_{\Psi}$ is at a distance of $\frac{1-r}{\sqrt{6}}$ from $E_{\Psi}$ and has a $\mu$ value of $\frac{1-r}{6}$, giving $\frac{2 \mu}{(1-4 \mu)}=\frac{1-r}{1+2 r}$. But $\mathcal{C}_{p}^{2}=1-r^{2} \geq(1-r)^{2}$ giving $\mathcal{C}_{p} \geq \frac{1-r}{1+2 r}$ with equality only for $r=0$. Hence, the points give a state that is entangled except for the case of $r=0$. The result directly contradicts a statement in the key 'theorem' of Ref. [14 the proof of which we fail to appreciate. Even taking only that maximally entangled as $E_{\Psi}(r=0)$ to get the $W_{\mu}(r=0)$ as a baseline does not work. While the perpendicular from $S_{\Psi}$ to $W_{\mu}(r=0)$ is right at the separable boundary, for another $W_{\mu}(r)$ from an $E_{\Psi}(r)$ between $S_{\Psi}$ and the maximally entangled one, i.e. 
also check that $D_{2}\left(W_{\mu}, W_{\frac{1}{4}}\right)=\frac{\sqrt{6}}{4} \nu_{1}$, and $\nu_{1}$ effectively measures the distance of $W_{\mu}$ from the maximally mixed state $W_{\frac{1}{4}}$.

The generalized Werner states for $x \in\left[-\frac{1}{3}, 0\right)$ can be simply analyzed by relaxing our condition of $\mu_{k}$ ordering. It is like taking the same analysis with an inverted ordering for the original two-qutetrait pure state construction, starting with $\sum_{k=0}^{n-1} \sqrt{\mu_{3-k}}\left|e_{k}\right\rangle\left|e_{k}^{\prime}\right\rangle$. It can easily be obtained that all such states are separable. It is not difficult to find the description of such states within our coordinate system. They are the $\mathcal{M}_{(31)}$ subspace with $\mu^{\prime} \equiv \mu_{0}=\mu_{1}=\mu_{2}, \mu_{3}=1-3 \mu^{\prime}$, with $\frac{1}{3} \geq \mu^{\prime} \geq \frac{1}{4}$. The only nonzero $\nu_{i}$ is $\nu_{3}=3\left(4 \mu^{\prime}-1\right)$, with the parameter $x$ as $1-4 \mu^{\prime}$ having the exact admissible range of value. Explicitly, we have $W_{\mu^{\prime}}=\left(1-4 \mu^{\prime}\right) E_{e_{3}}+\mu^{\prime} I$ where $E_{e_{3}} \equiv\left|e_{3}\right\rangle\left\langle e_{3}\right|$. The corresponding separability condition for $C_{p}^{\prime}$ being concurrence of the pure state $\left|e_{3}\right\rangle$ is $C_{p}^{\prime} \leq \frac{2 \mu^{\prime}}{4 \mu^{\prime}-1}$ trivially satisfied.

Actually, the above result for the separability of the $W_{\mu}$ and states implies that for any $x|\Phi\rangle\langle\Phi|+\frac{1-x}{4} I$ leading from the maximally mixed state at the center to an arbitrary pure state $E_{\Phi}$ on the sphere of radius $R_{\text {out }}=\frac{\sqrt{6}}{4}$ where the pure states reside, the separable states extended from the center to a distance $\frac{\sqrt{6}}{4} x$ of the value $\frac{\sqrt{6}}{4\left(2 \mathcal{C}_{p}+1\right)}$ as constrained by the concurrence $\mathcal{C}_{p}$ value of $E_{\Phi}$ as the pure state closest to the set of generalized Werner states.

\section{FINAL REMARKS}

We want to emphasize that our parametrization, for a generic system of mixed states as well as the modified form for a bipartite system, introduced is particularly interesting. As full coordination of the space of mixed states, many interesting quantities and geometric features of the space [1] have quite direct descriptions under the parameters. That is mostly the result of having the $\nu_{i}$, or hierarchical $\mu_{k}$, incorporated as part of the parameters/coordinates, giving the unambiguous identification of the subspaces of the pure states with the geometric result of Eq.(7). This is especially so for the case of the bipartite systems. For the twoqubit case, for example, the parametrization identifies the entanglement as independent of the four, or generally $4(N-1)$ for two-quNit, purely local parameters. Moreover, one

with generic $r \in(0,1)$, the intersecting point of that $W_{\mu}(r)$ and the perpendicular from $S_{\Psi}$ to $W_{\mu}(r=0)$ can be seen not to give a right distance to $W_{\frac{1}{4}}\left[\right.$ along $\left.W_{\mu}(r)\right]$ to agree with our separability results. A result like that of Ref.[14] could otherwise give a good base to obtain the full explicit characterization of the separability under our parametrization of the generic two-qubit state. 
has entanglements of the pure state parts in the eigenensemble, i.e. the $\mathcal{C}_{p}, \mathcal{C}_{\Psi_{i}}$, and $\mathcal{C}_{e_{i}}$, systematically identified. The intriguing plausibility of expressing entanglement for any mixed state in terms of them together $\nu_{i}$ is worth further studies.

While the analytically technical difficulty to get to a general parameterized complete description of any particular measure of entanglement for all possible mixed states is still a hurdle to surmount even for the two-qubit system, not to say a more complicated one, our parametrization scheme may have merits for the studies of entanglement features in various parts of the space of all such mixed states. Our study here focuses only on the two-qubit case, but many qualitative features of our results are expected to be somewhat applicable in a more general case. The role of the entanglement of different parts, of a pure or mixed state, in contributing to the entanglement results of the whole, we believe, is of interest both theoretically and experimentally. Our scheme for the parametrization may serve this kind of purpose well and similar analyses along the approach can be applied to other bipartite system with comparable results.

For two-qubit entanglement in particular, let us say a few words about the difficulty in getting fully complete results. With the parametrization of the general density matrix and hence its partial transpose, putting down the PPT condition is straightforward but still tedious and not very illustrative. For the partial transpose to be semi-positive, it requires all its principal minors to be nonnegative. That is $15-4=11$ inequalities. Even if the condition of a nonnegative determinant suffices, for example in the case of $c_{21}=0$, reading physics out of the inequality is quite nontrivial. To calculate the negativity or the concurrence is about solving the corresponding quartic characteristic polynomial. We fail to obtain a full analytical result. However, the latter may not be an impossible task. Moreover, some semi-analytic or numerical methods may be applied to help improve our understanding of the (more) general results. That is not only about appreciating the entanglement patterns of the mixed states. Because the parametrization is particularly well suited to illustrate the geometric structure of the full space of mixed states, the results may help to clarify further the relationship between the two, as some of our results do.

In looking at how the entanglement of the parts contribute to that of the whole state, we have essentially illustrated a good approach to identify such parts, and presented the results for the whole as interference or partial cancellation between those of the parts, for pure and nontrivial mixed states, respectively. In the interest of our general parametrization 
scheme, we have the eigenensemble mixing parameter $\mu_{k}$ restricted to have the hierarchy. For a more general application looking into some specific classes of states of a certain system, being the two-qubit one or otherwise, the basic approach and some of the results may be used interestingly without that hierarchy imposed.

\section{Appendix: The full two-qubit density matrix with our parametrization.}

— the bold-typed basis : $|\mathbf{0}\rangle \equiv\left|\phi \phi^{\prime}\right\rangle|\mathbf{1}\rangle \equiv\left|\phi_{\perp} \phi_{\perp}^{\prime}\right\rangle,|\mathbf{2}\rangle \equiv\left|\phi \phi_{\perp}^{\prime}\right\rangle,|\mathbf{3}\rangle \equiv\left|\phi_{\perp} \phi^{\prime}\right\rangle$.

$$
\begin{aligned}
& \rho_{\text {oo }}:\left(\mu_{0}-\mu_{3}\right) q_{+}^{2}+\left(\mu_{1}-\mu_{3}\right) q_{-}^{2}\left|c_{21}\right|^{2}+\left(\mu_{2}-\mu_{3}\right) q_{-}^{2}\left|c_{0}\right|^{2}\left|s_{21}\right|^{2}+\mu_{3} \\
& \rho_{01}:\left(\mu_{0}-\mu_{3}\right) e^{-i \zeta} q_{-} q_{+}-\left(\mu_{1}-\mu_{3}\right) e^{-i \zeta} q_{-} q_{+}\left|c_{21}\right|^{2}-\left(\mu_{2}-\mu_{3}\right) e^{-i \zeta} q_{-} q_{+}\left|c_{0}\right|^{2}\left|s_{21}\right|^{2} \\
& \rho_{02}:-\left(\mu_{1}-\mu_{3}\right) e^{-i \zeta} q_{-} c_{21} \bar{c}_{32} \bar{s}_{21}+\left(\mu_{2}-\mu_{3}\right) e^{-i \zeta} q_{-} c_{0} \bar{s}_{21}\left(\bar{c}_{0} c_{21} \bar{c}_{32}-\bar{s}_{0} s_{32}\right) \\
& \rho_{03}:-\left(\mu_{1}-\mu_{3}\right) e^{-i \zeta} q_{-} c_{21} \bar{s}_{32} \bar{s}_{21}+\left(\mu_{2}-\mu_{3}\right) e^{-i \zeta} q_{-} c_{0} \bar{s}_{21}\left(\bar{c}_{0} c_{21} \bar{s}_{32}+\bar{s}_{0} c_{32}\right) \\
& \rho_{10}:\left(\mu_{0}-\mu_{3}\right) e^{i \zeta} q_{-} q_{+}-\left(\mu_{1}-\mu_{3}\right) e^{i \zeta} q_{-} q_{+}\left|c_{21}\right|^{2}-\left(\mu_{2}-\mu_{3}\right) e^{i \zeta} q_{-} q_{+}\left|c_{0}\right|^{2}\left|s_{21}\right|^{2} \\
& \rho_{11}:\left(\mu_{0}-\mu_{3}\right) q_{-}^{2}+\left(\mu_{1}-\mu_{3}\right) q_{+}^{2}\left|c_{21}\right|^{2}+\left(\mu_{2}-\mu_{3}\right) q_{+}^{2}\left|c_{0}\right|^{2}\left|s_{21}\right|^{2}+\mu_{3} \\
& \rho_{12}:\left(\mu_{1}-\mu_{3}\right) q_{+} c_{21} \bar{c}_{32} \bar{s}_{21}-\left(\mu_{2}-\mu_{3}\right) q_{+} c_{0} \bar{s}_{21}\left(\bar{c}_{0} c_{21} \bar{c}_{32}-\bar{s}_{0} s_{32}\right) \\
& \rho_{13}:\left(\mu_{1}-\mu_{3}\right) q_{+} c_{21} \bar{s}_{32} \bar{s}_{21}-\left(\mu_{2}-\mu_{3}\right) q_{+} c_{0} \bar{s}_{21}\left(\bar{c}_{0} c_{21} \bar{s}_{32}+\bar{s}_{0} c_{32}\right) \\
& \rho_{20}:-\left(\mu_{1}-\mu_{3}\right) e^{i \zeta} q_{-} \bar{c}_{21} c_{32} s_{21}+\left(\mu_{2}-\mu_{3}\right) e^{i \zeta} q_{-} \bar{c}_{0} s_{21}\left(c_{0} \bar{c}_{21} c_{32}-s_{0} \bar{s}_{32}\right) \\
& \rho_{21}:\left(\mu_{1}-\mu_{3}\right) q_{+} \bar{c}_{21} c_{32} s_{21}-\left(\mu_{2}-\mu_{3}\right) q_{+} \bar{c}_{0} s_{21}\left(c_{0} \bar{c}_{21} c_{32}-s_{0} \bar{s}_{32}\right) \\
& \rho_{22}:\left(\mu_{1}-\mu_{3}\right)\left|c_{32}\right|^{2}\left|s_{21}\right|^{2}+\left(\mu_{2}-\mu_{3}\right)\left|c_{0} \bar{c}_{21} c_{32}-s_{0} \bar{s}_{32}\right|^{2}+\mu_{3} \\
& \rho_{23}:\left(\mu_{1}-\mu_{3}\right)\left|s_{21}\right|^{2} c_{32} \bar{s}_{32}+\left(\mu_{2}-\mu_{3}\right)\left(\bar{c}_{0} c_{21} \bar{s}_{32}+\bar{s}_{0} c_{32}\right)\left(c_{0} \bar{c}_{21} c_{32}-s_{0} \bar{s}_{32}\right) \\
& \rho_{30}:-\left(\mu_{1}-\mu_{3}\right) e^{i \zeta} q_{-} \bar{c}_{21} s_{32} s_{21}+\left(\mu_{2}-\mu_{3}\right) e^{i \zeta} q_{-} \bar{c}_{0} s_{21}\left(c_{0} \bar{c}_{21} s_{32}+s_{0} \bar{c}_{32}\right) \\
& \rho_{31}:\left(\mu_{1}-\mu_{3}\right) q_{+} \bar{C}_{21} s_{32} s_{21}-\left(\mu_{2}-\mu_{3}\right) q_{+} \bar{C}_{0} s_{21}\left(c_{0} \bar{c}_{21} s_{32}+s_{0} \bar{c}_{32}\right) \\
& \rho_{32}:\left(\mu_{1}-\mu_{3}\right)\left|s_{21}\right|^{2} \bar{c}_{32} s_{32}+\left(\mu_{2}-\mu_{3}\right)\left(c_{0} \bar{c}_{21} s_{32}+s_{0} \bar{c}_{32}\right)\left(\bar{c}_{0} c_{21} \bar{c}_{32}-\bar{s}_{0} s_{32}\right) \\
& \rho_{33}:\left(\mu_{1}-\mu_{3}\right)\left|s_{21}\right|^{2}\left|s_{32}\right|^{2}+\left(\mu_{2}-\mu_{3}\right)\left|c_{0} \bar{c}_{21} s_{32}+s_{0} \bar{c}_{32}\right|^{2}+\mu_{3}
\end{aligned}
$$

\section{Acknowledgments :}

The work is supported by the research grants number 109-2112-M-008-016 and 1102112-M-008-016 of the MOST of Taiwan. 
Data availability : the theoretical research reported involves no data.

[1] Bengtsson, I. , Życzkowski, K. : Geometry of Quantum States. Cambridge University Press, Cambridge (2006)

[2] Nielsen, M., Chuang, I. : Quantum Computation and Quantum Information. Cambridge University Press, New York (2000)

[3] Horodecki, R., Horodecki, P., Horodecki, M., Horodecki, K. Quantum entanglement. Rev. Mod. Phys. 81, 865-942 (2009).

[4] Werner, R. F. : Quantum states with Einstein-Podolski-Rosen correlations admitting a hiddenvariable model. Phys. Rev. A 40, 4277-4281 (1989).

[5] Bruening, E., Makela, H., Messina, A., Petruccione, F. : Parametrizations of density matrices. J. Mod. Opt. 59, 1-20 (2012).

[6] Vidal, G., Tarrach, R. : Robustness of entanglement. Phys. Rev. A 59, 141-155 (1999).

[7] Peres, A. : Separability criterion for density matrices. Phys. Rev. Lett. 77, 1413-1415 (1996).

[8] Horodecki, M., Horodecki, P., Horodecki, R. : Separability of mixed states: Necessary and sufficient conditions. Phys. Lett. A 223, 1-8 (1996).

[9] Życzkowski, K., Horodecki, P., Sanpera, A., Lewenstein, M. : Volume of the set of separable states. Phys. Rev. A 58, 883-892(1998)

[10] Vidal, G., Werner, R. F. : A computable measure of entanglement. Phys. Rev. A 65, 032314 (2002)

[11] Wootters, W. K. : Entanglement of formation of an arbitrary state of two qubits. Phys. Rev. Lett. $80,2245-2248$ (1998)

[12] Verstraete, F., Audenaert, K., Dehaene, J., DeMoor, B. : A comparison of the entanglement measures negativity and concurrence. J. Phys. A 34, 10327-10332 (2001)

[13] Miranowicz, A., Grudka, A. : Ordering two-qubit states with concurrence and negativity. Phys. Rev. A 70, 032326 (2004)

[14] Lockhart, R. B., Steiner, M. J. : Preserving entanglement under perturbation and sandwiching all separable states. Phys. Rev. A 65, 022107 (2002) 